\title{
On the Inviscid Limit of the 3D Navier-Stokes Equations with Generalized Navier-Slip Boundary Conditions
}

\author{
Yuelong Xiao • Zhouping Xin
}

Received: 3 June 2013 / Accepted: 8 July 2013 / Published online: 26 July 2013

(C) School of Mathematical Sciences, University of Science and Technology of China and Springer-Verlag Berlin Heidelberg 2013

\begin{abstract}
In this paper, we investigate the vanishing viscosity limit problem for the 3-dimensional (3D) incompressible Navier-Stokes equations in a general bounded smooth domain of $R^{3}$ with the generalized Navier-slip boundary conditions $u^{\varepsilon} \cdot n=$ $0, n \times\left(\omega^{\varepsilon}\right)=\left[B u^{\varepsilon}\right]_{\tau}$ on $\partial \Omega$. Some uniform estimates on rates of convergence in $C\left([0, T], L^{2}(\Omega)\right)$ and $C\left([0, T], H^{1}(\Omega)\right)$ of the solutions to the corresponding solutions of the ideal Euler equations with the standard slip boundary condition are obtained.
\end{abstract}

Keywords Navier-Stokes equations $\cdot$ Slip boundary conditions $\cdot$ Inviscid limit

Mathematics Subject Classification (2010) 35Q30 - 76D05

\section{Introduction}

Let $\Omega \subset R^{3}$ be a bounded smooth domain. We consider the Navier-Stokes equations

$$
\begin{aligned}
& \partial_{t} u^{\varepsilon}-\varepsilon \Delta u^{\varepsilon}+u^{\varepsilon} \cdot \nabla u^{\varepsilon}+\nabla p^{\varepsilon}=0, \quad \text { in } \Omega, \\
& \nabla \cdot u^{\varepsilon}=0, \quad \text { in } \Omega
\end{aligned}
$$

and the Euler equations

$$
\partial_{t} u+u \cdot \nabla u+\nabla p=0, \quad \text { in } \Omega,
$$

Y. Xiao

School of Mathematics and Computational Science, Xiangtan University, Xiangtan, China

Y. Xiao · Z. Xin $(\bowtie)$

The Institute of Mathematical Sciences, The Chinese University of Hong Kong, New Territories, Hong Kong

e-mail: zpxin@ims.cuhk.edu.hk 


$$
\nabla \cdot u=0, \quad \text { in } \Omega \text {. }
$$

We are interested in the vanishing viscosity limit problem: do the solutions of the Navier-Stokes equations (1.1), (1.2) converge to those of the Euler equation (1.3) with the same initial data as $\varepsilon$ vanishes?

The vanishing viscosity limit problem for the Navier-Stokes equations is a classical issue and has been well studied when the domain has no boundaries, various convergence results have been obtained, see for instances [12, 14, 15, 19, 20, 27, 35].

However, in the presence of a physical boundary, the situation is much more complicated, and the problem becomes challenging due to the boundary layers. This is so even in the case that the corresponding solution to the initial boundary value problem of the Euler equations remains smooth.

Indeed, in the presence of a boundary $\partial \Omega$, one of the most important physical boundary conditions for the Euler equations (1.3) is the following slip boundary condition:

$$
u \cdot n=0 \quad \text { on } \partial \Omega
$$

and the initial boundary value problem of the Euler equations (1.3) with the slip boundary condition (1.5) has a smooth solution at least locally in time [10, 22]. Corresponding to the slip boundary condition (1.5) for the Euler equations, there are different choices of boundary conditions for the Navier-Stokes equations, one is the mostly used no-slip boundary condition, i.e.,

$$
u^{\varepsilon}=0, \quad \text { on } \Omega \text {. }
$$

This Dirichlet type boundary condition was proposed by Stokes ([34]) assuming that fluid particles are adherent to the boundary due to the positive viscosity. Although the well-posedness of the smooth solution to the initial boundary value problem of the Navier-Stokes equations with the no-slip boundary condition can be established quite easily (at least locally in time), the asymptotic convergence of the solution to the corresponding solution of the Euler equations (1.3) with the boundary condition (1.5) as the viscosity coefficient $\varepsilon$ tends to zero is one of major open problems except special cases (see $[13,29,32,33,45]$ ) due to the possible appearance of the boundary layers. In general, only some sufficient conditions are obtained for the convergence in $L^{2}(\Omega)$, see $[21,24,38]$. However, there are some new results in the $2 \mathrm{D}$ case, announced recently in [26].

Another class of boundary conditions for the Navier-Stokes equations is the Navier-slip boundary conditions, i.e.,

$$
u^{\varepsilon} \cdot n=0,\left[2\left(S\left(u^{\varepsilon}\right) n\right)+\gamma u^{\varepsilon}\right]_{\tau}=0, \quad \text { on } \partial \Omega,
$$

where $2 S\left(u^{\varepsilon}\right)=\left(\nabla u^{\varepsilon}+\left(\nabla u^{\varepsilon}\right)^{T}\right)$ is the viscous stress tensor, and $\gamma$ is a smooth given function. Such conditions were proposed by Navier in [30], which allow the fluid to slip at the boundary, and have important applications for problems with rough boundaries, prorated boundaries, and interfacial boundary problems, see $[1,8,31$, 37]. The well-posedness of the Navier-Stokes equations (1.1), (1.2) with the Navierslip boundary conditions and related results have been established, see $[8,43]$ and the references therein. 
In a recent paper [16], the Navier-slip boundary condition (1.7) is written to the following generalized one:

$$
u^{\varepsilon} \cdot n=0,\left[2\left(S\left(u^{\varepsilon}\right) n\right)+A u^{\varepsilon}\right]_{\tau}=0, \quad \text { on } \partial \Omega,
$$

with $A$ is a smooth symmetric tensor. The well-posedness of the Navier-Stokes equations (1.1), (1.2) with the generalized Navier-slip boundary condition is indeed similar to the classical one.

On the other hand, the following vorticity-slip boundary condition:

$$
u^{\varepsilon} \cdot n=0, n \times\left(\omega^{\varepsilon}\right)=\beta u^{\varepsilon}, \quad \text { on } \partial \Omega,
$$

with $\beta$ is a smooth function is introduced in [43], where $\omega=\nabla \times u$ is the vorticity of the fluid.

It is noticed that

$$
(2(S(v) n)-(\nabla \times v) \times n)_{\tau}=G D(v)_{\tau}, \quad \text { on } \partial \Omega,
$$

where $G D(v)=-2 S(n) v$ (see [43]). Hence the generalized slip condition (1.8) is equivalent to the following generalized vorticity-slip condition:

$$
u^{\varepsilon} \cdot n=0, n \times\left(\omega^{\varepsilon}\right)=\left[B u^{\varepsilon}\right]_{\tau} \quad \text { on } \partial \Omega
$$

with $B$ a given smooth symmetric tensor on the boundary. The equivalence can also be seen for instance in $[2-4,7,39,41]$.

Compared to the case of no-slip boundary condition, the asymptotic behavior of solutions to the Navier-Stokes equations with the Navier-slip boundary conditions as $\varepsilon \rightarrow 0$ is relatively easy to establish. Some strong convergence results in 2D (see $[11,25])$ and various weak convergence results in $3 \mathrm{D}$ (see $[16,17,23]$ ) are also established, where it has been shown that

$$
\left\|u^{\varepsilon}-u\right\|^{2}+\varepsilon \int_{0}^{t}\left\|u^{\varepsilon}-u\right\|_{1}^{2} d t \leq c \varepsilon^{\frac{3}{2}}
$$

and

$$
\left\|u^{\varepsilon}-u\right\|_{L^{\infty}([0, T] \times \Omega)} \leq c \varepsilon^{\frac{3}{8}(1-s)}
$$

for some $s>0$, see [16] for details.

For the homogeneous case, i.e., $B=0$ in (1.11), better convergence results are available. The $H^{3}(\Omega)$ convergence and $H^{2}(\Omega)$ estimate on the rate of convergence are obtained in [41] for flat domains. These results are improved to $W^{k, p}(\Omega)$ in $[3$, 4]. However, such a strong convergence cannot be expected for general domains, except the case that the initial vorticity vanishes on the boundary [42]. Note that the convergence in $H^{2}(\Omega)$ or, $W^{k, p}(\Omega)$ (for $k \geq 2$ ), implies that the limiting solution of the Euler equations satisfies the boundary condition (1.11) with $B=0$.

However, it is shown in $[5,6]$ that the solution to the Euler equations with the slip boundary condition (1.5) cannot satisfy the extra condition $n \times \omega=0$ on the boundary in general, and would satisfy $n \times \omega=0$ on $\partial \Omega$ only if $\omega \cdot n=0$ on $\partial \Omega$. 
This implies that the only possible case for convergence of solutions to the NavierStokes equations in $H^{2}(\Omega)$ is the one obtained in [42], where the following estimate on the convergence rate:

$$
\left\|u^{\varepsilon}-u\right\|^{2}+\varepsilon\left\|u^{\varepsilon}-u\right\|_{1}^{2}+\varepsilon \int_{0}^{t}\left(\left\|u^{\varepsilon}-u\right\|_{1}^{2}+\varepsilon\left\|u^{\varepsilon}-u\right\|_{2}^{2}\right) d t \leq c \varepsilon^{2}
$$

is obtained. Furthermore, a $W^{s, p}(\Omega)$ convergence result can also be obtained in this case, see [9].

For general initial data and domains in the homogeneous case, i.e., $B=0$ in (1.11), the best estimate on the rate of convergence established so far is in $W^{1, p}(\Omega)$ in [40], which implies in the particular case $p=2$ that

$$
\left\|u^{\varepsilon}-u\right\|^{2}+\varepsilon\left\|u^{\varepsilon}-u\right\|_{1}^{2} \leq c \varepsilon^{2-s}
$$

with $s=\frac{1}{2}$, where $u^{\varepsilon}$ and $u$ are solutions to (1.1), (1.2), (1.9) and (1.3), (1.5), respectively.

However, all the strong convergence results depend essentially the homogeneous property, i.e., $n \times \omega=0$ on $\partial \Omega$. Indeed, for general $B$ (not identically equal to zero on $\partial \Omega$ ), there is no estimate on the rate of convergence in $H^{k}(\Omega)$ (or $W^{k, p}(\Omega)$ ) for $k \geq 1$, as far as the authors are aware. It should be noted that the co-normal uniform estimates have been established in [28] for general domains and general Navier-slip boundary conditions, which guarantee the uniform boundness estimates of solutions to the Navier-Stokes equations and the Euler equations in $W^{1, \infty}(\Omega)$. Yet, some additional efforts are still needed to obtain an estimate on the rate of convergence as in (1.14).

In a recent paper [44], we proposed the following slip boundary condition:

$$
u^{\varepsilon} \cdot n=0, \quad \omega^{\varepsilon} \cdot n=0, \quad n \times\left(\Delta u^{\varepsilon}\right)=0 \quad \text { on } \partial \Omega
$$

for the Navier-Stokes equations (1.1), (1.2) and obtained the following estimate on the rate of convergence:

$$
\left\|u^{\varepsilon}-u\right\|_{1}^{2}+\varepsilon\left\|u^{\varepsilon}-u\right\|_{2}^{2}+\varepsilon \int_{0}^{t}\left(\left\|u^{\varepsilon}-u\right\|_{2}^{2}+\varepsilon\left\|u^{\varepsilon}-u\right\|_{3}^{2}\right) d t \leq c \varepsilon^{2-s}
$$

for any $s>0$.

In this paper, we investigate the vanishing viscosity problem for the Navier-Stokes equations (1.1), (1.2) on a general bounded smooth domain $\Omega \subset R^{3}$ with the generalized vorticity-slip boundary condition (1.11). Our main result is the following estimate on the rate of convergence for the solutions:

Theorem 1.1 Let $u(t)$ be the smooth solution to the boundary value problem of Euler equations (1.3), (1.4), (1.5) on $[0, T]$ with $u(0)=u_{0} \in H^{3}(\Omega)$ which satisfies the corresponding assumptions on the initial data in Theorem 1 of [28] (see also Lemma 2.1 in next section), and $u^{\varepsilon}(t)$ be the solution to the boundary value problem 
of the Navier-Stokes equations (1.1), (1.2), (1.11) with the same initial data $u_{0}$. Then, there is $T_{0}>0$ such that

$$
\left\|u^{\varepsilon}-u\right\|^{2}+\varepsilon \int_{0}^{T_{1}}\left\|u^{\varepsilon}-u\right\|_{1}^{2} d t \leq c \varepsilon^{2-s}, \quad \text { on }\left[0, T_{0}\right]
$$

and

$$
\left\|u^{\varepsilon}-u\right\|_{1}^{2}+\varepsilon \int_{0}^{T_{1}}\left\|u^{\varepsilon}-u\right\|_{2}^{2} d t \leq c \varepsilon^{1-s}, \quad \text { on }\left[0, T_{0}\right]
$$

for any $s>0$ and $\varepsilon$ small enough. Consequently,

$$
\left\|u^{\varepsilon}-u\right\|_{1, p}^{p} \leq c_{p} \varepsilon^{1-s}, \quad \text { on }\left[0, T_{0}\right]
$$

for $2 \leq p<\infty$, any $s>0$ and $\varepsilon$ small enough, and

$$
\left\|u^{\varepsilon}-u\right\|_{L^{\infty}([0, T] \times \Omega)} \leq c \varepsilon^{\frac{2}{5}\left(1-s^{\prime}\right)}
$$

for any $s^{\prime}>0$.

(1.19) yields the desired estimate on the rate of convergence of the solutions of the Navier-Stokes equations to that of the Euler equations in $C\left([0, T], H^{1}(\Omega)\right)$ norm for general domains with general Navier-slip boundary conditions. It should be noted that the estimates (1.18) and (1.21) improve (1.12) and (1.13), and (1.19), (1.20) are even better than (1.15) stated in [40] for the special case $B=0$. Indeed, the estimate (1.19) is optimal in the sense that $s$ cannot be taken to be 0 due to boundary layers in general, see Remark 3.1 below for details.

These estimates are motivated by our recent work [44], where the first order derivative estimates for the solutions are obtained for the Navier-Stokes equations with a new vorticity boundary condition (1.16), and the work of Masmoudi and Rousset in [28], where uniform estimates on $\left\|u^{\varepsilon}\right\|_{W^{1 . \infty}}$ for the solutions to the NavierStokes equations with the general Navier-slip boundary condition are obtained.

Our approach is an elementary energy estimate for the difference of the solutions between the Navier-Stokes equations and the Euler equations. Some suitable integrating by part formulas in terms of the vorticity are successfully used to get the optimal rate of convergence. In comparison to the asymptotic analysis method associate to the boundary layers (see $[16,18,23,40]$ ), we should not need any correctors near the boundary. Meanwhile, the uniform regularity obtained in [28] plays an essential role in our analysis.

The rest of the paper is organized as follows: In the next section, we give a preliminary on the well-posedness of the initial boundary value problem of the NavierStokes equations (1.1), (1.2) and (1.11), and the local uniform regularity of the solutions. Then, the estimates on the asymptotic convergence (1.18), (1.19), (1.20) and (1.21) are established in Sect. 3. 


\section{Preliminaries}

Let $\Omega \subset R^{3}$ be a general bounded smooth domain. We begin by considering the following Stokes problem:

$$
\begin{aligned}
& \alpha u-\Delta u+\nabla q=f, \quad \text { in } \Omega, \\
& \nabla \cdot u=0, \quad \text { in } \Omega, \\
& u \cdot n=0, \quad n \times(\nabla \times u)=[B u]_{\tau}, \quad \text { on } \partial \Omega,
\end{aligned}
$$

where $B$ is smooth symmetric tensor. Set

$$
\begin{aligned}
& H=\left\{u \in L^{2}(\Omega) ; \nabla \cdot u=0, \text { in } \Omega ; u \cdot n=0 ; \text { on } \partial \Omega\right\}, \\
& V=H^{1}(\Omega) \cap H, \\
& W=\left\{u \in H^{2}(\Omega) ; n \times(\nabla \times u)=[B u]_{\tau} \text { on, } \partial \Omega\right\} .
\end{aligned}
$$

It is well known that for any $v \in V$, one has

$$
\|v\|_{1} \leq c\|\nabla \times v\|
$$

Note that

$$
[B u]_{\tau} \cdot \phi=B u \cdot \phi
$$

for any $\phi$ satisfying $\phi \cdot n=0$ on the boundary. We associate the Stokes problem (2.1)-(2.3) with the following bilinear form:

$$
a_{\alpha}(u, \phi)=\alpha(u, \phi)+\int_{\partial \Omega} B u \cdot \phi+\int_{\Omega}(\nabla \times u) \cdot(\nabla \times \phi) .
$$

Note that

$$
\left|\int_{\partial \Omega} B u \cdot u\right| \leq c\|u\|\|u\|_{1}
$$

for all $u \in V$ and (2.4). It follows that $a_{\alpha}(u, \phi)$ is a positive definite symmetric bilinear form if $\alpha$ large enough, and is closed with the domain $\mathcal{D}\left(a_{\alpha}\right)=V$. Similar to the discussions in [43], one can define the self-adjoint operator associated with $a_{\alpha}$ as

$$
A=\alpha I-P \Delta
$$

with the domain $\mathcal{D}(A)=V \cap W$, which implies

$$
\|v\|_{2} \leq c\|v\|+\|P \Delta v\|, \quad \forall v \in \mathcal{D}(A) .
$$

Now, we turn to the boundary value problem of the Navier-Stokes equations (1.1), (1.2) on $\Omega \subset R^{3}$ with the generalized vorticity-slip boundary condition (1.11). 
By using the Galerkin method based on the orthogonal eigenvectors of $A$, noting the energy equation

$$
\frac{1}{2} \frac{d}{d t}\left\|u^{\varepsilon}\right\|^{2}+\varepsilon\left\|\nabla \times u^{\varepsilon}\right\|^{2}+\varepsilon \int_{\partial \Omega} B u^{\varepsilon} \cdot u^{\varepsilon}=0
$$

valid for approximate solutions, and (2.4), (2.6), one can obtain the global existence of weak solutions to the boundary value problem of Navier-Stokes equations (1.1), (1.2), (1.11) (see the corresponding definition in, for instance, [43]).

Denoting the energy equation

$$
\frac{1}{2} \frac{d}{d t}\left(\left\|\nabla \times u^{\varepsilon}\right\|^{2}+\int_{\partial \Omega} B u^{\varepsilon} \cdot u^{\varepsilon}\right)+\varepsilon\left\|P \Delta u^{\varepsilon}\right\|^{2}+\left(u^{\varepsilon} \cdot \nabla u^{\varepsilon}, P \Delta u^{\varepsilon}\right)=0
$$

for approximate solutions, (2.4), and (2.6)-(2.8), one can show that if $u_{0}^{\varepsilon} \in V$, then there is a maximum time interval $\left[0, T^{\varepsilon}\right)$ such that the weak solutions are the unique strong one on $\left[0, T^{\varepsilon}\right.$ ) (see the corresponding definition in, for instance, [43]).

It follows from Lemma 3.10 in [43] that

$$
(2(S(u) n)-\omega \times n)_{\tau}=G D(u)_{\tau}
$$

with $G D(u)=-2 S(n) u$.

Then, the boundary conditions (1.7)-(1.9) can be written to (1.11), and (1.8) is equivalent to (1.11). Hence, one has the following uniform regularity result for the solutions to the Navier-Stokes equations:

Lemma 2.1 (Masmoudi and Rousset [28]) Let $m>6$ be an integer and $\Omega$ be a $C^{m+2}$ domain. Consider $u_{0} \in E^{m} \cap H$ such that $\nabla u_{0} \in W_{\mathrm{co}}^{1, \infty}$. Then there is $T_{m}>0$ such that for all sufficient small $\varepsilon$, there is a unique solution $u^{\varepsilon} \in C\left(\left[0, T_{m}\right], E^{m}\right)$ to the Navier-Stokes problem (1.1), (1.2), (1.11) with $u^{\varepsilon}(0)=u_{0}$. Moreover, there is a constant $C$ such that

$$
\left\|u^{\varepsilon}\right\|_{H_{\mathrm{co}}^{m}(\Omega)}+\left\|\nabla u^{\varepsilon}\right\|_{H_{\mathrm{co}}^{m-1}(\Omega)}+\left\|\nabla u^{\varepsilon}\right\|_{W_{\mathrm{co}}^{1, \infty}(\Omega)}+\varepsilon \int_{0}^{t}\left\|\nabla^{2} u^{\varepsilon}\right\|_{H_{\mathrm{co}}^{m-1}(\Omega)}^{2} d t \leq C
$$

on $\left[0, T_{m}\right]$.

Here $H_{\mathrm{co}}^{m}(\Omega)$ and $W_{\mathrm{co}}^{1, \infty}(\Omega)$ are co-norm vector-spaces, $\|\cdot\|_{H_{\mathrm{co}}^{m}(\Omega)}$ and $\|\cdot\|_{W_{\mathrm{co}}^{1, \infty}(\Omega)}$ denote the corresponding norms, and

$$
E^{m}=\left\{u \in H_{\mathrm{co}}^{m}(\Omega) \mid \nabla u \in H_{\mathrm{co}}^{m-1}(\Omega)\right\} .
$$

Since the notations are rather too complicated to be expressed, we omit it here and refer to Masmoudi and Rousset [28] for the details. This uniform regularity implies in particular the following uniform bound:

$$
\left\|u^{\varepsilon}\right\|_{W^{1, \infty}} \leq C, \quad \text { on }\left[0, T_{m}\right]
$$

which plays an essential role in our estimates. 


\section{Convergence of the Solutions}

We now turn to the purpose of this paper to establish the convergence with a rate for the solutions $u^{\varepsilon}$ to $u$. We start with the basic $L^{2}$-estimate.

Theorem 3.1 Let $u_{0} \in H^{3}(\Omega)$ satisfy the assumptions stated in Theorem 1.1 and $u(t)$ be the solution to the Euler equations (1.3), (1.4), (1.5) on $[0, T]$ with $u(0)=u_{0}$, and $u(t)=u^{\varepsilon}(t)$ be the solution to the Navier-Stokes problem (1.1), (1.2), (1.11) with $u^{\varepsilon}(0)=u_{0}$. Then

$$
\left\|u^{\varepsilon}-u\right\|^{2}+\varepsilon \int_{0}^{T_{0}}\left\|u^{\varepsilon}-u\right\|_{1}^{2} d t \leq c \varepsilon^{2-s} \quad \text { on }\left[0, T_{0}\right]
$$

for any $s>0$ and $\varepsilon$ small enough, where $T_{0}=\min \left\{T, T_{m}\right\}$. Consequently,

$$
\left\|u^{\varepsilon}-u\right\|_{L^{\infty}([0, T] \times \Omega)} \leq c \varepsilon^{\frac{2}{5}\left(1-s^{\prime}\right)}
$$

for any $s^{\prime}>0$.

Proof Note that $u^{\varepsilon}-u$ satisfies

$$
\begin{aligned}
& \partial_{t}\left(u^{\varepsilon}-u\right)-\varepsilon \Delta\left(u^{\varepsilon}-u\right)+\Phi+\nabla\left(p^{\varepsilon}-p\right)=\varepsilon \Delta u, \quad \text { in } \Omega, \\
& \nabla \cdot u^{\varepsilon}=0, \quad \text { in } \Omega, \\
& \left(u^{\varepsilon}-u\right) \cdot n=0, \quad n \times\left(\omega^{\varepsilon}-\omega\right)=\left[B\left(u^{\varepsilon}-u\right)+B u\right]_{\tau}-n \times \omega, \quad \text { on } \partial \Omega,
\end{aligned}
$$

where $\omega=\nabla \times u, \omega^{\varepsilon}=\nabla \times u^{\varepsilon}$,

$$
\Phi=u \cdot \nabla\left(u^{\varepsilon}-u\right)+\left(u^{\varepsilon}-u\right) \cdot \nabla u+\left(u^{\varepsilon}-u\right) \cdot \nabla\left(u^{\varepsilon}-u\right) .
$$

Then, the following identity holds:

$$
\frac{1}{2} \frac{d}{d t}\left\|u^{\varepsilon}-u\right\|^{2}+\varepsilon\left\|\nabla \times\left(u^{\varepsilon}-u\right)\right\|^{2}+\mathcal{B}_{0}+\left(\Phi, u^{\varepsilon}-u\right)=\varepsilon\left(\Delta u, u^{\varepsilon}-u\right)
$$

where

$$
\mathcal{B}_{0}=\varepsilon \int_{\partial \Omega} n \times\left(\omega^{\varepsilon}-\omega\right)\left(u^{\varepsilon}-u\right)=\varepsilon \int_{\partial \Omega}\left(B\left(u^{\varepsilon}-u\right)+B u-n \times \omega\right)\left(u^{\varepsilon}-u\right) .
$$

Note that

$$
\begin{aligned}
\int_{\partial \Omega} n \times\left(\omega^{\varepsilon}-\omega\right)\left(u^{\varepsilon}-u\right) & =\int_{\partial \Omega}\left(B\left(u^{\varepsilon}-u\right)+B u-n \times \omega\right)\left(u^{\varepsilon}-u\right) \\
& \leq c \int_{\partial \Omega}\left(\left|\left(u^{\varepsilon}-u\right)\right|^{2}+\left|\left(u^{\varepsilon}-u\right)\right|\right) \\
& \leq c\left(\left\|u^{\varepsilon}-u\right\|\left\|\omega^{\varepsilon}-\omega\right\|+\left|u^{\varepsilon}-u\right|_{L^{1}(\partial \Omega)}\right) .
\end{aligned}
$$


Here and in what follows, $|f|_{L^{p}(\partial \Omega)}$ denotes the standard $L^{p}$-norm of $\mathrm{f}$ on the boundary $\partial \Omega$ for any $p>0$. It follows from the trace theorem that

$$
\left|u^{\varepsilon}-u\right|_{L^{1}(\partial \Omega)} \leq c\left|u^{\varepsilon}-u\right|_{L^{q}(\partial \Omega)} \leq c\left\|u^{\varepsilon}-u\right\|_{H^{s}(\Omega)}
$$

for any $q>1$ and then any $s>0$.

By interpolation (see [36]), we have

$$
\left\|u^{\varepsilon}-u\right\|_{H^{s}(\Omega)} \leq c\left\|u^{\varepsilon}-u\right\|^{(1-s)}\left\|u^{\varepsilon}-u\right\|_{1}^{s} \leq c\left\|u^{\varepsilon}-u\right\|^{(1-s)}\left\|\omega^{\varepsilon}-\omega\right\|^{s} .
$$

Note that

$$
\varepsilon\left\|u^{\varepsilon}-u\right\|^{(1-s)}\left\|\omega^{\varepsilon}-\omega\right\|^{s} \leq \delta \varepsilon\left\|\omega^{\varepsilon}-\omega\right\|^{2}+c_{\delta}\left(\left\|u^{\varepsilon}-u\right\|^{2}+\varepsilon^{2-s}\right)
$$

for any $s \in(0,1)$ and

$$
\varepsilon\left\|u^{\varepsilon}-u\right\|\left\|\omega^{\varepsilon}-\omega\right\| \leq \varepsilon^{2}\left\|\omega^{\varepsilon}-\omega\right\|^{2}+\left\|u^{\varepsilon}-u\right\|^{2} .
$$

Then, we have

$$
\mathcal{B}_{0} \leq 2 \delta \varepsilon\left\|\omega^{\varepsilon}-\omega\right\|^{2}+c_{\delta}\left\|u^{\varepsilon}-u\right\|^{2}+\varepsilon^{2-s}
$$

for any $s \in(0,1)$ and $\varepsilon$ small enough.

Note also that

$$
\left(\Phi, u^{\varepsilon}-u\right)=\left(\left(u^{\varepsilon}-u\right) \cdot \nabla u, u^{\varepsilon}-u\right) \leq c\left\|u^{\varepsilon}-u\right\|^{2}
$$

and

$$
\varepsilon\left(\Delta u, u^{\varepsilon}-u\right) \leq\left\|u^{\varepsilon}-u\right\|^{2}+c \varepsilon^{2} .
$$

It follows that

$$
\frac{d}{d t}\left\|u^{\varepsilon}-u\right\|^{2}+\varepsilon\left\|\nabla \times\left(u^{\varepsilon}-u\right)\right\|^{2} \leq c\left(\left\|u^{\varepsilon}-u\right\|^{2}+\varepsilon^{2-s}\right)
$$

for any $s \in(0,1)$, and then for any $s>0$ and $\varepsilon$ small enough. Note that $u^{\varepsilon}(0)-$ $u(0)=0$. Then, (3.1) follows from (3.10) and the Gronwall lemma.

Consequently, by using the Gagliardo-Nirenberg interpolation inequality, one has

$$
\left\|u^{\varepsilon}-u\right\|_{L^{\infty}(\Omega)} \leq c\left\|u^{\varepsilon}-u\right\|^{\frac{2}{5}}\left\|u^{\varepsilon}-u\right\|_{W^{1, \infty}(\Omega)}^{\frac{3}{5}} \leq c \varepsilon^{\frac{2}{5}\left(1-s^{\prime}\right)}
$$

for any $s^{\prime}>0$. The theorem is proved.

Next, we prove the major estimate in this paper.

Theorem 3.2 Let $u_{0} \in H^{3}(\Omega)$ satisfies the assumptions stated in Theorem 1.1, and $u(t)$ be the solution to the Euler equations (1.3), (1.4), (1.5) on $[0, T]$ with $u(0)=u_{0}$, 
and $u(t)=u^{\varepsilon}(t)$ be the solution to the Navier-Stokes problem (1.1), (1.2), (1.11) with $u^{\varepsilon}(0)=u_{0}$. Then

$$
\left\|u^{\varepsilon}-u\right\|_{1}^{2}+\varepsilon \int_{0}^{T}\left\|u^{\varepsilon}-u\right\|_{2}^{2} d t \leq c \varepsilon^{1-s}, \quad \text { on }\left[0, T_{0}\right]
$$

for any $s>0$ and $\varepsilon$ small enough, where $T_{0}=\min \left\{T, T_{m}\right\}$.

Proof Let $s$ be the same as in Theorem 3.1. Note the smoothness of the solutions and

$$
\partial_{t}\left(u^{\varepsilon}-u\right) \cdot n=0, \quad \text { on } \partial \Omega .
$$

It follows from (3.3)-(3.5) that

$$
\begin{aligned}
& \frac{1}{2} \frac{d}{d t}\left\|\left(\omega^{\varepsilon}-\omega\right)\right\|^{2}+\varepsilon\left\|P \Delta\left(u^{\varepsilon}-u\right)\right\|^{2}-\left(\Phi, P \Delta\left(u^{\varepsilon}-u\right)\right) \\
& \quad=\int_{\partial \Omega} \partial_{t}\left(u^{\varepsilon}-u\right) \cdot\left(n \times\left(\omega^{\varepsilon}-\omega\right)\right)-\varepsilon\left(\Delta u, P \Delta\left(u^{\varepsilon}-u\right)\right)
\end{aligned}
$$

where $P$ is the Lerray projection,

$$
\Phi=u \cdot \nabla\left(u^{\varepsilon}-u\right)+\left(u^{\varepsilon}-u\right) \cdot \nabla u+\left(u^{\varepsilon}-u\right) \cdot \nabla\left(u^{\varepsilon}-u\right)
$$

and that

$$
\begin{aligned}
& \int_{\partial \Omega} \partial_{t}\left(u^{\varepsilon}-u\right) \cdot\left(n \times\left(\omega^{\varepsilon}-\omega\right)\right) \\
& \quad=\int_{\partial \Omega} \partial_{t}\left(u^{\varepsilon}-u\right) \cdot\left(B\left(u^{\varepsilon}-u\right)+B u-n \times \omega\right) \\
& \quad=\frac{1}{2} \frac{d}{d t}\left(\int_{\partial \Omega} B\left(u^{\varepsilon}-u\right) \cdot\left(u^{\varepsilon}-u\right)+2 \int_{\partial \Omega}\left(u^{\varepsilon}-u\right) \cdot(B u-n \times \omega)\right)-\mathcal{B}_{1}
\end{aligned}
$$

where

$$
\mathcal{B}_{1}=\int_{\partial \Omega}\left(u^{\varepsilon}-u\right) \cdot \partial_{t}(B u-n \times \omega) .
$$

It follows from Theorem 3.1 and (3.9) that

$$
\left|\mathcal{B}_{1}\right| \leq c \int_{\partial \Omega}\left|\left(u^{\varepsilon}-u\right)\right| \leq \delta\left\|\left(\omega^{\varepsilon}-\omega\right)\right\|^{2}+c \varepsilon^{1-s} .
$$

Note also that

$$
\varepsilon\left|\left(\Delta u, P \Delta\left(u^{\varepsilon}-u\right)\right)\right| \leq \frac{\varepsilon}{2}\left\|P \Delta\left(u^{\varepsilon}-u\right)\right\|^{2}+c \varepsilon
$$

and that

$$
-\left(\Phi, P \Delta\left(u^{\varepsilon}-u\right)\right)=\left(P \Phi,-\Delta\left(u^{\varepsilon}-u\right)\right)
$$




$$
\begin{aligned}
& =\left(\nabla \times \Phi,\left(\omega^{\varepsilon}-\omega\right)\right)+\int_{\partial \Omega} n \times\left(\omega^{\varepsilon}-\omega\right) \cdot P \Phi \\
& =\left(\nabla \times \Phi,\left(\omega^{\varepsilon}-\omega\right)\right)+\int_{\partial \Omega}\left(B\left(u^{\varepsilon}-u\right)+B u-n \times \omega\right) \cdot P \Phi .
\end{aligned}
$$

Then, we get

$$
\frac{1}{2} \frac{d}{d t} E+\frac{\varepsilon}{2}\left\|P \Delta\left(u^{\varepsilon}-u\right)\right\|^{2} \leq N+B N+B N L+c\left(\left\|\left(\omega^{\varepsilon}-\omega\right)\right\|^{2}+\varepsilon^{1-s}\right)
$$

for $\varepsilon$ small enough, where

$$
\begin{aligned}
& E=\left\|\left(\omega^{\varepsilon}-\omega\right)\right\|^{2}-\left(\int_{\partial \Omega} B\left(u^{\varepsilon}-u\right) \cdot\left(u^{\varepsilon}-u\right)+2 \int_{\partial \Omega}\left(u^{\varepsilon}-u\right) \cdot(B u-n \times \omega)\right) \\
& N=\left|\left(\nabla \times \Phi,\left(\omega^{\varepsilon}-\omega\right)\right)\right| \\
& B N=\left|\int_{\partial \Omega} B\left(u^{\varepsilon}-u\right) \cdot P \Phi\right| \\
& B N L=\left|\int_{\partial \Omega}(B u-n \times \omega) \cdot P \Phi\right| .
\end{aligned}
$$

The term $N$ can be estimated easily by using the Sobolev inequalities and the known uniform bounds for $\left\|u^{\varepsilon}\right\|_{\infty}$ and $\left\|\nabla u^{\varepsilon}\right\|_{\infty}$. The term $B N$ can be estimated after integrating by part properly. While, the estimate for the leading order term $B N L$ is rather complicated due to the possible appearance of boundary layers. We now carry out these estimates.

Estimates on $N$ :

The term $N$ is estimated as follows.

Note that

$$
\begin{aligned}
\nabla \times \Phi & =\nabla \times\left(\omega \times\left(u^{\varepsilon}-u\right)+\left(\omega^{\varepsilon}-\omega\right) \times u+\left(\omega^{\varepsilon}-\omega\right) \times\left(u^{\varepsilon}-u\right)\right) \\
& =\left[\omega, u^{\varepsilon}-u\right]+\left[\omega^{\varepsilon}-\omega, u\right]+\left[\omega^{\varepsilon}-\omega, \omega^{\varepsilon}-\omega\right]
\end{aligned}
$$

where

$$
[\varphi, \psi]=\psi \cdot \nabla \varphi-\varphi \cdot \nabla \psi
$$

Also

$$
\left|\left(\left(u^{\varepsilon}-u\right) \cdot \nabla \omega, \omega^{\varepsilon}-\omega\right)\right| \leq c\left\|\omega^{\varepsilon}-\omega\right\|^{2}
$$

and

$$
\left|\left(\omega \cdot \nabla\left(u^{\varepsilon}-u\right), \omega^{\varepsilon}-\omega\right)\right| \leq c\left\|u^{\varepsilon}-u\right\|_{1}\left\|\omega^{\varepsilon}-\omega\right\| \leq c\left\|\omega^{\varepsilon}-\omega\right\|^{2} .
$$

It follows that

$$
\left|\left(\left[\omega, u^{\varepsilon}-u\right], \omega^{\varepsilon}-\omega\right)\right| \leq c\left\|\omega^{\varepsilon}-\omega\right\|^{2} .
$$


On the other hand,

$$
\begin{aligned}
& \left(u \cdot \nabla\left(\omega^{\varepsilon}-\omega\right), \omega^{\varepsilon}-\omega\right)=0 \\
& \left(\left(\omega^{\varepsilon}-\omega\right) \nabla u, \omega^{\varepsilon}-\omega\right) \leq c\left\|\omega^{\varepsilon}-\omega\right\|^{2} .
\end{aligned}
$$

It follows that

$$
\left|\left(\left[\omega^{\varepsilon}-\omega, u\right], \omega^{\varepsilon}-\omega\right)\right| \leq c\left\|\omega^{\varepsilon}-\omega\right\|^{2}
$$

Note that

$$
\left(\left(u^{\varepsilon}-u\right) \cdot \nabla\left(\omega^{\varepsilon}-\omega\right), \omega^{\varepsilon}-\omega\right)=0
$$

and

$$
\begin{aligned}
& \left(\left(\omega^{\varepsilon}-\omega\right) \cdot \nabla\left(u^{\varepsilon}-u\right), \omega^{\varepsilon}-\omega\right) \\
& \quad \leq\left\|\nabla\left(u^{\varepsilon}-u\right)\right\|_{\infty}\left\|\omega^{\varepsilon}-\omega\right\|^{2} \leq c\left\|\omega^{\varepsilon}-\omega\right\|^{2}
\end{aligned}
$$

(here and below we will use the uniform regularity that $\left\|\nabla u^{\varepsilon}\right\|_{\infty} \leq c$ ). It follows that

$$
\left|\left(\left[\omega^{\varepsilon}-\omega, u^{\varepsilon}-u\right], \omega^{\varepsilon}-\omega\right)\right| \leq c\left\|\omega^{\varepsilon}-\omega\right\|^{2} .
$$

Hence

$$
N \leq c\left\|\omega^{\varepsilon}-\omega\right\|^{2}
$$

Estimates on $B N$ :

Next, we estimate the term

$$
B N=\left|\int_{\partial \Omega} B\left(u^{\varepsilon}-u\right) \cdot P \Phi\right| .
$$

Note that

$$
P \Phi=\Phi+\nabla p_{\Phi}
$$

involves a scalar function $p_{\Phi}$, which is difficult to estimate on the boundary. We will transform it to an estimate on $\Omega$ by integrating by parts.

To this end, we first extend $n, B$ to the interior of $\Omega$ as follows:

$$
n(x)=\varphi(r(x)) \nabla(r(x))
$$

and

$$
B(x)=\varphi(r(x)) B(\Pi x),
$$

where

$$
r(x)=\min _{y \in \partial \Omega} d(x, y)
$$

and

$$
\Pi x=y_{x} \in \partial \Omega,
$$


such that

$$
r(x)=d\left(x, y_{x}\right)
$$

which is uniquely defined on $\Omega_{\sigma}=\{x \in \Omega, r(x) \leq 2 \sigma\}$ for some $\sigma>0$, and $\varphi(s)$ is smooth and compactly supported in $[0,2 \sigma)$ such that

$$
\varphi(0)=1, \quad \text { on }[0, \sigma] .
$$

Then, we can deduce the estimate of the boundary term $B N$ to an interior estimate on $\Omega$ by the Stokes formula

$$
\begin{aligned}
\int_{\partial \Omega} B\left(u^{\varepsilon}-u\right) \cdot P \Phi & =\int_{\partial \Omega}\left(n \times B\left(u^{\varepsilon}-u\right) \cdot(n \times P \Phi)\right) \\
& =\left(n \times B\left(u^{\varepsilon}-u\right), \nabla \times \Phi\right)-\left(\nabla \times\left(n \times B\left(u^{\varepsilon}-u\right)\right), P \Phi\right)
\end{aligned}
$$

since $P \Phi \cdot n=0$ on the boundary, and $\nabla \times P \Phi=\nabla \times \Phi$.

Note that

$$
\left|\left(n \times B\left(u^{\varepsilon}-u\right),\left(u^{\varepsilon}-u\right) \cdot \nabla \omega\right)\right| \leq c\left\|u^{\varepsilon}-u\right\|^{2} \leq c\left\|\omega^{\varepsilon}-\omega\right\|^{2}
$$

and

$$
\left|\left(n \times B\left(u^{\varepsilon}-u\right), \omega \cdot \nabla\left(u^{\varepsilon}-u\right)\right)\right| \leq c\left\|u^{\varepsilon}-u\right\|_{1}^{2} \leq c\left\|\omega^{\varepsilon}-\omega\right\|^{2} .
$$

It follows that

$$
\left|\left(n \times B\left(u^{\varepsilon}-u\right),\left[\omega, u^{\varepsilon}-u\right]\right)\right| \leq c\left\|\omega^{\varepsilon}-\omega\right\|^{2} .
$$

Note that $u \cdot n=0$ on $\partial \Omega, \nabla \cdot u=0$. Then

$$
\begin{aligned}
& \left|\left(n \times B\left(u^{\varepsilon}-u\right), u \cdot \nabla\left(\omega^{\varepsilon}-\omega\right)\right)\right| \\
& \quad=\left|\left(\omega^{\varepsilon}-\omega, u \cdot \nabla\left(n \times B\left(u^{\varepsilon}-u\right)\right)\right)\right| \leq c\left\|u^{\varepsilon}-u\right\|_{1}^{2} \leq c\left\|\omega^{\varepsilon}-\omega\right\|^{2}
\end{aligned}
$$

and

$$
\left|\left(n \times B\left(u^{\varepsilon}-u\right),\left(\omega^{\varepsilon}-\omega\right) \cdot \nabla u\right)\right| \leq c\left\|\omega^{\varepsilon}-\omega\right\|^{2} .
$$

It follows that

$$
\left|\left(n \times B\left(u^{\varepsilon}-u\right),\left[\omega^{\varepsilon}-\omega, u\right]\right)\right| \leq c\left\|\omega^{\varepsilon}-\omega\right\|^{2} .
$$

Similarly

$$
\begin{aligned}
& \left|\left(n \times B\left(u^{\varepsilon}-u\right),\left(u^{\varepsilon}-u\right) \cdot \nabla\left(\omega^{\varepsilon}-\omega\right)\right)\right| \\
& \quad=\left|\left(\omega^{\varepsilon}-\omega,\left(u^{\varepsilon}-u\right) \cdot \nabla\left(n \times B\left(u^{\varepsilon}-u\right)\right)\right)\right| \\
& \quad \leq c\left\|u^{\varepsilon}-u\right\|_{1}^{2} \leq c\left\|\omega^{\varepsilon}-\omega\right\|^{2}
\end{aligned}
$$

and

$$
\left|\left(n \times B\left(u^{\varepsilon}-u\right),\left(\omega^{\varepsilon}-\omega\right) \cdot \nabla\left(u^{\varepsilon}-u\right)\right)\right| \leq c\left\|\omega^{\varepsilon}-\omega\right\|^{2} .
$$


It follows that

$$
\left|\left(n \times B\left(u^{\varepsilon}-u\right),\left[\omega^{\varepsilon}-\omega, u^{\varepsilon}-u\right]\right)\right| \leq c\left\|\omega^{\varepsilon}-\omega\right\|^{2} .
$$

Hence

$$
\left|\left(n \times B\left(u^{\varepsilon}-u\right), \nabla \times \Phi\right)\right| \leq c\left\|\omega^{\varepsilon}-\omega\right\|^{2} .
$$

Note also that

$$
\begin{aligned}
\left|\left(\nabla \times\left(n \times B\left(u^{\varepsilon}-u\right)\right), P \Phi\right)\right| & \leq\left\|\nabla \times\left(n \times B\left(u^{\varepsilon}-u\right)\right)\right\|\|\Phi\| \\
& \left.\leq c \|\left(u^{\varepsilon}-u\right)\right)\left\|_{1}^{2} \leq c\right\| \omega^{\varepsilon}-\omega \|^{2} .
\end{aligned}
$$

Hence, we have

$$
B N \leq c\left\|\omega^{\varepsilon}-\omega\right\|^{2} .
$$

Estimate on $\int_{\partial \Omega}(B u-n \times \omega) \cdot P \Phi$ :

Finally, we estimate the leading order term on the boundary

$$
B N L=\left|\int_{\partial \Omega}(B u-n \times \omega) \cdot P \Phi\right| .
$$

It should be noted that the estimate is trivial if

$$
[B u]_{\tau}-n \times \omega=0
$$

which is the condition that the solution to the ideal Euler system satisfies the same boundary condition (1.11) just as that the solution to the Navier-Stokes system does so there are no strong boundary layers. This applies to the cases treated in [41, 42]. Here, $[B u]_{\tau}-n \times \omega$ may be not equal zero, then boundary layers may occur. Additional efforts are needed to overcome the new difficulties.

Similar to the above, we have

$$
\begin{aligned}
\left|\int_{\partial \Omega}(B u-n \times \omega) \cdot P \Phi\right|= & \left|\int_{\partial \Omega} n \times(B u-n \times \omega) \cdot n \times P \Phi\right| \\
= & \mid(n \times(B u-n \times \omega), \nabla \times \Phi) \\
& -(\nabla \times(n \times(B u-n \times \omega)), P \Phi) \mid .
\end{aligned}
$$

The term $|(n \times(B u-n \times \omega), \nabla \times \Phi)|$ is relatively easy to estimate since $\nabla \times \Phi$ does not involve the pressure function.

Rewrite $\Phi$ as

$$
\Phi=\Phi_{1}+\Phi_{2}+\Phi_{3}
$$

where

$$
\begin{aligned}
& \Phi_{1}=\left(u^{\varepsilon}-u\right) \cdot \nabla u \\
& \Phi_{2}=u \cdot \nabla\left(u^{\varepsilon}-u\right)
\end{aligned}
$$




$$
\Phi_{3}=\left(u^{\varepsilon}-u\right) \cdot \nabla\left(u^{\varepsilon}-u\right)
$$

Note that

$$
\begin{aligned}
& \left|\left(n \times(B u-n \times \omega), \nabla \times \Phi_{1}\right)\right| \\
& =\left|\left(n \times(B u-n \times \omega), \nabla \times\left(\left(u^{\varepsilon}-u\right) \cdot \nabla u\right)\right)\right| \\
& =\mid \int_{\partial \Omega} n \times\left(\left(u^{\varepsilon}-u\right) \cdot \nabla u\right) \cdot(n \times(B u-n \times \omega)) \\
& \quad+\left(\nabla \times(n \times(B u-n \times \omega)),\left(u^{\varepsilon}-u\right) \cdot \nabla u\right) \mid \\
& \quad \leq c\left(\int_{\partial \Omega}\left|u^{\varepsilon}-u\right|+\left\|u^{\varepsilon}-u\right\|\right) \leq c\left(\left\|\omega^{\varepsilon}-\omega\right\|^{2}+\varepsilon^{1-s}\right)
\end{aligned}
$$

and

$$
\begin{aligned}
& \left|\left(n \times(B u-n \times \omega), \nabla \times \Phi_{3}\right)\right| \\
& =\left|\left(n \times(B u-n \times \omega), \nabla \times\left(\left(u^{\varepsilon}-u\right) \cdot \nabla\left(u^{\varepsilon}-u\right)\right)\right)\right| \\
& =\mid \int_{\partial \Omega} n \times\left(\left(u^{\varepsilon}-u\right) \cdot \nabla\left(u^{\varepsilon}-u\right)\right) \cdot(n \times(B u-n \times \omega)) \\
& \quad+\left(\nabla \times(n \times(B u-n \times \omega)),\left(u^{\varepsilon}-u\right) \cdot \nabla\left(u^{\varepsilon}-u\right)\right) \mid \\
& \quad \leq c\left(\int_{\partial \Omega}\left|u^{\varepsilon}-u\right|+\left\|u^{\varepsilon}-u\right\|_{1}^{2}\right) \leq c\left(\left\|\omega^{\varepsilon}-\omega\right\|^{2}+\varepsilon^{1-s}\right)
\end{aligned}
$$

since $u^{\varepsilon}-u$ is smooth, so that

$$
\left|\nabla\left(u^{\varepsilon}-u\right)\right| \leq c\left\|\nabla\left(u^{\varepsilon}-u\right)\right\|_{\infty} \leq c, \quad \text { on } \partial \Omega .
$$

To estimate $\left(n \times(B u-n \times \omega), \nabla \times \Phi_{2}\right)$, we note

$$
\nabla \times(\psi \cdot \nabla \phi)=\psi \cdot \nabla(\nabla \times \phi)+\nabla \psi^{\perp} \cdot \nabla \phi
$$

where $\nabla \psi^{\perp}$ is expressed in component by

$$
\left(\nabla \psi^{\perp} \cdot \nabla \phi\right)_{j}=(-1)^{j+1} \partial_{j+1} \psi \cdot \nabla \phi_{j+1}+(-1)^{j+2} \partial_{j+2} \psi \cdot \nabla \phi_{j+2}
$$

with the index modulated by 3 . Hence, we have

$$
\begin{aligned}
& \left|\left(n \times(B u-n \times \omega), \nabla \times \Phi_{2}\right)\right| \\
& \quad=\left|\left(n \times(B u-n \times \omega), \nabla \times\left(u \cdot \nabla\left(u^{\varepsilon}-u\right)\right)\right)\right| \\
& \quad=\left|\left(n \times(B u-n \times \omega), u \cdot \nabla\left(\omega^{\varepsilon}-\omega\right)\right)+\left(n \times(B u-n \times \omega), \nabla u^{\perp} \cdot \nabla\left(u^{\varepsilon}-u\right)\right)\right| .
\end{aligned}
$$


Note that

$$
\begin{aligned}
& \left|\left(n \times(B u-n \times \omega), u \cdot \nabla\left(\omega^{\varepsilon}-\omega\right)\right)\right| \\
& =\left|\left(\omega^{\varepsilon}-\omega, u \cdot \nabla(n \times(B u-n \times \omega))\right)\right| \\
& =\left(\nabla \times\left(u^{\varepsilon}-u\right), u \cdot \nabla(n \times(B u-n \times \omega))\right) \\
& =\mid \int_{\partial \Omega} n \times\left(u^{\varepsilon}-u\right) \cdot(u \cdot \nabla(n \times(B u-n \times \omega))) \\
& \quad+\left(u^{\varepsilon}-u, \nabla \times(u \cdot \nabla(n \times(B u-n \times \omega)))\right) \mid \\
& \quad \leq c\left(\int_{\partial \Omega}\left|u^{\varepsilon}-u\right|+\left\|u^{\varepsilon}-u\right\|\right) \leq c\left(\left\|\omega^{\varepsilon}-\omega\right\|^{2}+\varepsilon^{1-s}\right) .
\end{aligned}
$$

Note also that

$$
\begin{aligned}
\left(\partial_{j} u\right. & \left.\cdot \nabla\left(u^{\varepsilon}-u\right)_{j},(n \times(B u-n \times \omega))_{k}\right) \\
= & \left(\partial_{j} u, \nabla\left(\left(u^{\varepsilon}-u\right)_{j}(n \times(B u-n \times \omega))_{k}\right)\right. \\
\quad & \left.\quad\left(\partial_{j} u \cdot \nabla(n \times(B u-n \times \omega))_{k},\left(u^{\varepsilon}-u\right)_{j}\right)\right),
\end{aligned}
$$

and that

$$
\begin{aligned}
& \left|\left(\partial_{j} u, \nabla\left(\left(u^{\varepsilon}-u\right)_{j}(n \times(B u-n \times \omega))_{k}\right)\right)\right| \\
& \quad=\left|\int_{\partial \Omega}\left(u^{\varepsilon}-u\right)_{j}(n \times(B u-n \times \omega))_{k} \partial_{j} u \cdot n\right| \\
& \quad \leq c\|u\|_{1, \infty}^{2} \int_{\partial \Omega}\left|u^{\varepsilon}-u\right| \leq c \int_{\partial \Omega}\left|u^{\varepsilon}-u\right| \leq c\left(\left\|\omega^{\varepsilon}-\omega\right\|^{2}+\varepsilon^{1-s}\right)
\end{aligned}
$$

since $\nabla \cdot \partial_{j} u=0$, and that

$$
\left|\left(\partial_{j} u \cdot \nabla(n \times(B u-n \times \omega))_{k},\left(u^{\varepsilon}-u\right)_{j}\right)\right| \leq c\left\|u^{\varepsilon}-u\right\| \leq c \varepsilon^{1-s}
$$

it follows that

$$
\left|\left(n \times(B u-n \times \omega), \nabla \times \Phi_{2}\right)\right| \leq c\left(\left\|\omega^{\varepsilon}-\omega\right\|^{2}+\varepsilon^{1-s}\right) .
$$

Then, we get

$$
|(n \times(B u-n \times \omega), \nabla \times \Phi)| \leq c\left(\left\|\omega^{\varepsilon}-\omega\right\|^{2}+\varepsilon^{1-s}\right) .
$$

It remains to estimate $|(\nabla \times(n \times(B u-n \times \omega)), P \Phi)|$. There is also a difficulty arising from $P \Phi$. To do it, it is noticed that

$$
P \Phi=u \cdot \nabla\left(u^{\varepsilon}-u\right)-\left(u^{\varepsilon}-u\right) \cdot \nabla u+P \tilde{\Phi}
$$


where

$$
\tilde{\Phi}=2\left(u^{\varepsilon}-u\right) \cdot \nabla u+\left(u^{\varepsilon}-u\right) \cdot \nabla\left(u^{\varepsilon}-u\right)
$$

with

$$
u \cdot \nabla\left(u^{\varepsilon}-u\right)-\left(u^{\varepsilon}-u\right) \cdot \nabla u=\nabla \times\left(\left(u^{\varepsilon}-u\right) \times u\right) \in H
$$

since that $\left(u^{\varepsilon}-u\right) \cdot n=0$ and $u \cdot n=0$ on $\partial \Omega$ will imply

$$
\left(u^{\varepsilon}-u\right) \times u=\lambda n
$$

and

$$
H=\left\{v=\nabla \times \phi ; \phi \in H^{1}(\Omega), n \times \phi=0\right\} .
$$

Hence, we have

$$
P\left(u \cdot \nabla\left(u^{\varepsilon}-u\right)-\left(u^{\varepsilon}-u\right) \cdot \nabla u\right)=u \cdot \nabla\left(u^{\varepsilon}-u\right)-\left(u^{\varepsilon}-u\right) \cdot \nabla u
$$

so that

$$
\begin{aligned}
(\nabla \times & (n \times(B u-n \times \omega)), P \Phi) \\
= & \left(\nabla \times(n \times(B u-n \times \omega)), u \cdot \nabla\left(u^{\varepsilon}-u\right)\right. \\
& \left.\quad-\left(u^{\varepsilon}-u\right) \cdot \nabla u\right)+(\nabla \times(n \times(B u-n \times \omega)), P \tilde{\Phi})
\end{aligned}
$$

and

$$
\|P \tilde{\Phi}\| \leq c\|\tilde{\Phi}\| \leq c\left(\|u\|_{1, \infty}+\left\|u^{\varepsilon}-u\right\|_{1, \infty}\right)\left\|u^{\varepsilon}-u\right\| \leq c\left\|u^{\varepsilon}-u\right\| \leq c \varepsilon^{1-s} .
$$

These properties help us to complete the rest of the estimates.

First, we have

$$
|(\nabla \times(n \times(B u-n \times \omega)), P \tilde{\Phi})| \leq c\|P \tilde{\Phi}\| \leq c\|\tilde{\Phi}\| \leq c\left\|u^{\varepsilon}-u\right\| \leq c \varepsilon^{1-s} .
$$

Next, note that

$$
\begin{aligned}
& \left|\left(\nabla \times(\times(B u-n \times \omega)), u \cdot \nabla\left(u^{\varepsilon}-u\right)\right)\right| \\
& \quad=\left|\left(u^{\varepsilon}-u, u \cdot \nabla(\nabla \times(\times(B u-n \times \omega)))\right)\right| \leq c\|u\|_{3}^{2}\left\|u^{\varepsilon}-u\right\| \leq c \varepsilon^{1-s}
\end{aligned}
$$

since $\nabla u=0$ in $\Omega$ and $u \cdot n=0$ on $\partial \Omega$.

Note also that

$$
\left|\left(\nabla \times(n \times(B u-n \times \omega)),\left(u^{\varepsilon}-u\right) \nabla u\right)\right| \leq c\|u\|_{2}^{2}\left\|u^{\varepsilon}-u\right\| \leq c \varepsilon^{1-s} .
$$

Hence, we have

$$
|(\nabla \times(n \times(B u-n \times \omega)), P \Phi)| \leq c \varepsilon^{1-s} .
$$


Then, we conclude that

$$
B N L=\left|\int_{\partial \Omega}(B u-n \times \omega) \cdot P \Phi\right| \leq c\left(\left\|\omega^{\varepsilon}-\omega\right\|^{2}+\varepsilon^{1-s}\right) .
$$

In conclusion, it follows from (3.13), (3.14), (3.16) and (3.20) that

$$
\frac{1}{2} \frac{d}{d t} E+\frac{\varepsilon}{2}\left\|P \Delta\left(u^{\varepsilon}-u\right)\right\|^{2} \leq c\left(\left\|\omega^{\varepsilon}-\omega\right\|^{2}+\varepsilon^{1-s}\right) .
$$

Recall that

$$
\begin{aligned}
& E=\left\|\left(\omega^{\varepsilon}-\omega\right)\right\|^{2}-\left(\int_{\partial \Omega} B\left(u^{\varepsilon}-u\right) \cdot\left(u^{\varepsilon}-u\right)+2 \int_{\partial \Omega}\left(u^{\varepsilon}-u\right) \cdot(B u-n \times \omega)\right) \\
&\left|\int_{\partial \Omega} B\left(u^{\varepsilon}-u\right) \cdot\left(u^{\varepsilon}-u\right)\right| \leq c \int_{\partial \Omega}\left|\left(u^{\varepsilon}-u\right)\right|^{2} \\
& \leq c\left\|u^{\varepsilon}-u\right\|\left\|\omega^{\varepsilon}-\omega\right\| \leq\left\|\omega^{\varepsilon}-\omega\right\|^{2}+c\left\|u^{\varepsilon}-u\right\|^{2}
\end{aligned}
$$

and

$$
\left|\int_{\partial \Omega}\left(u^{\varepsilon}-u\right) \cdot(B u-n \times \omega)\right| \leq c \int_{\partial \Omega}\left|\left(u^{\varepsilon}-u\right)\right| \leq\left\|\omega^{\varepsilon}-\omega\right\|^{2}+c\left\|u^{\varepsilon}-u\right\|^{2} .
$$

It follows that

$$
\left\|\omega^{\varepsilon}-\omega\right\|^{2}+\varepsilon c \int_{0}^{t}\left\|P \Delta\left(u^{\varepsilon}-u\right)\right\|^{2} \leq c \int_{0}^{t}\left\|\omega^{\varepsilon}-\omega\right\|^{2}+c \varepsilon^{1-s} .
$$

By using the Gronwall Lemma, one gets

$$
\left\|\omega^{\varepsilon}-\omega\right\|^{2} \leq c \varepsilon^{1-s}
$$

on $\left[0, T_{0}\right]$ for any given $s>0$ and $\varepsilon$ for small enough. Then

$$
\left\|u^{\varepsilon}-u\right\|_{1}^{2} \leq c \varepsilon^{1-s}
$$

and

$$
\varepsilon \int_{0}^{t}\left\|P \Delta\left(u^{\varepsilon}-u\right)\right\|^{2} \leq c \varepsilon^{1-s} .
$$

Combining this with (2.7) and Theorem 3.1 implies that

$$
\varepsilon \int_{0}^{t}\left\|\left(u^{\varepsilon}-u\right)\right\|_{2}^{2} \leq c \varepsilon^{1-s}
$$

Note that

$$
\left\|\nabla\left(u^{\varepsilon}-u\right)\right\|_{p}^{p} \leq c\left\|\nabla\left(u^{\varepsilon}-u\right)\right\|_{\infty}^{p-2}\left\|\nabla\left(u^{\varepsilon}-u\right)\right\|^{2} .
$$


It follows that

$$
\left\|\nabla\left(u^{\varepsilon}-u\right)\right\|_{p}^{p} \leq c \varepsilon^{1-s}
$$

The theorem is proved.

Remark 3.1 The estimate (1.19) is optimal in the sense that $s$ cannot be taken to be 0 . Since if $s=0$, then (1.19) will imply that $u^{\varepsilon}-u$ is uniform bounded in $L^{2}\left(0, T ; H^{2}(\Omega)\right)$. Then, there is a subsequence such that $u^{\varepsilon_{n}}-u$ weakly convergence in $H^{2}(\Omega)$ for a.e. $t \in[0, T]$. Note that $u^{\varepsilon_{n}}-u$ strongly convergence to 0 in $H^{1}(\Omega)$ for all $t \in[0, T]$. It follows that

$$
u^{\varepsilon_{n}} \rightarrow u \quad \text { weakly in } H^{2}(\Omega) \text {, a.e. } t .
$$

Note that $W$ is closed in $H^{2}(\Omega)$, then is weakly closed. Hence, $u \in W$ for a.e. $t \in$ $[0, T]$, i.e.,

$$
[B u]_{\tau}-n \times \omega=0, \quad \text { a.e. } t .
$$

Since $u$ is smooth, then it holds for all $t \in[0, T]$. This is impossible in general, see $[5,6]$.

Acknowledgements This research is supported in part by NSFC 10971174, and Zheng Ge Ru Foundation, and Hong Kong RGC Earmarked Research Grants CUHK-4041/11P, CUHK-4042/08P, a Focus Area Grant from the Chinese University of Hong Kong, and a grant from Croucher Foundation.

\section{References}

1. Beavers, G.S., Joseph, D.D.: Boundary conditions at a naturally permeable wall. J. Fluid Mech. 30, 197-207 (1967)

2. Beirão da Veiga, H., Berselli, L.C.: Navier-Stokes equations: Greens matrices, vorticity direction, and regularity up to the boundary. J. Differ. Equ. 246(2), 597-628 (2009)

3. Beirão da Veiga, H., Crispo, F.: Sharp inviscid limit results under Navier type boundary conditions. An $L_{p}$ theory. J. Math. Fluid Mech. 12, 397-411 (2010)

4. Beirão da Veiga, H., Crispo, F.: Concerning the $W^{k, p}$-inviscid limit for 3D flows under a slip boundary condition. J. Math. Fluid Mech. 13, 117-135 (2011)

5. Beirão da Veiga, H., Crispo, F.: The 3D inviscid limit result under slip boundary conditions. A negative answer. J. Math. Fluid Mech. 14, 55-59 (2012)

6. Beirão da Veiga, H., Crispo, F.: A missed persistence property for the Euler equations and its effect on inviscid limits. Nonlinearity 25, 1661-1669 (2012)

7. Bellout, H., Neustupa, J., Penel, P.: On a $v$ continous family of strong solution to the Euler or NavierStokes equations with the Navier type boundary condition. Discrete Contin. Dyn. Syst. 27(4), 13531373 (2010)

8. Berselli, L.C.: Some results on the Navier-Stokes equations with Navier boundary conditions. Riv. Math. Univ. Parma (N. S.) 1(1), 1-75 (2010)

9. Berselli, L.C., Spirito, S.: On the vanishing viscosity limit of 3D Navier-Stokes equations under slip boundary conditions in general domains. Commun. Math. Phys.

10. Bourguignon, J.P., Brezis, H.: Remarks on the Euler equation. J. Funct. Anal. 15, 341-363 (1974)

11. Clopeau, T., Mikelić, A., Robert, R.: On the vanishing viscosity limit for the $2 \mathrm{D}$ incompressible Navier-Stokes equations with the friction type boundary conditions. Nonlinearity 11, 1625-1636 (1998)

12. Constantin, P.: Note on loss of regularity for solutions of the 3-D incompressible Euler and related equations. Commun. Math. Phys. 104, 311-326 (1986) 
13. Constantin, P.: On the Euler equations of incompressible fluids. Bull., New Ser., Am. Math. Soc. 44(4), 603-621 (2007)

14. Constantin, P., Foias, C.: Navier Stokes Equation. Univ. of Chicago Press, Chicago (1988)

15. Ebin, D.G., Marsden, J.: Groups of diffeomorphisms and the notion of an incompressible fluid. Ann. Math. 92, 102-163 (1970)

16. Gie, G.M., Kelliher, J.P.: Boundary layer analysis of the Navier-Stokes equations with generalized Navier boundary conditions. J. Differ. Equ. 253(6), 1862-1892 (2012)

17. Iftimie, D., Planas, G.: Inviscid limits for the Navier-Stokes equations with Navier friction boundary conditions. Nonlinearity 19(4), 899-918 (2006)

18. Iftimie, D., Sueur, F.: Viscous boundary layers for the Navier-Stokes equations with the Navier slip conditions. Arch. Ration. Mech. Anal. 199(1), 145-175 (2011)

19. Kato, T.: Nonstationary flows of viscous and ideal fluids in R3. J. Funct. Anal. 9, 296-305 (1972)

20. Kato, T.: Quasi-linear equations of evolution, with applications to partial differential equations. In: Spectral Theory and Differential Equations (Proc. Sympos., Dundee, 1974; Dedicated to Konrad Jörgens). Lecture Notes in Math., vol. 448, pp. 25-70. Springer, Berlin (1975)

21. Kato, T.: Remarks on zero viscosity limit for non-stationary Navier-Stokes flows with boundary. In: Chen, S.S. (ed.) Seminar on PDE, pp. 85-98. Springer, New York (1984)

22. Kato, T., Lai, C.Y.: Nonlinear evolution equations and the Euler flow. J. Funct. Anal. 56(1), 15-28 (1984)

23. Kelliher, J.: Navier-Stokes equations with Navier boundary conditions for a bounded domain in the plane. SIAM J. Math. Anal. 38(1), 210-232 (2006) (electronic)

24. Kelliher, J.: On Kato's conditions for vanishing viscosity. Indiana Univ. Math. J. 56(4), 1711-1721 (2007)

25. Lions, J.-L.: Quelques Méthodes de Résolution des Problèmes aux Limites non Linéires. Dunod, Paris (1969)

26. Maekawa, Y.: On the inviscid limit problem of the vorticity equations for viscous incompressible flows in the half plane. Preprint (2012)

27. Masmoudi, N.: Remarks about the inviscid limit of the Navier-Stokes system. Commun. Math. Phys. 270(3), 777-788 (2007)

28. Masmoudi, N., Rousset, F.: Uniform regularity for the Navier-Stokes equation with Navier boundary condition. Arch. Ration. Mech. Anal. 203, 529-575 (2012)

29. Mazzucato, A.: On the zero viscosity limit in incompressible fluids. Phys. Scr. 132, 0140002 (2008)

30. Navier, C.L.M.H.: Sur les lois de l'équilibre et du mouvement des corps élastiques. Mém. Acad. Sci. Inst. Fr. 6, 369 (1827)

31. Prizjev, N.V., Troian, S.M.: Influence of periodic wall roughness on the slip behaviour at liquid/solid interfaces. J. Fluid Mech. 554, 25-46 (2006)

32. Sammartino, M., Caflisch, R.E.: Zero viscosity limit for analytic solutions of the Navier-Stokes equation on a half-space. I. Existence for Euler and Prandtl equations. Commun. Math. Phys. 192(2), 433-461 (1998)

33. Sammartino, M., Caflisch, R.E.: Zero viscosity limit for analytic solutions of the Navier-Stokes equation on a half-space. II. Construction of the Navier-Stokes solution. Commun. Math. Phys. 192(2), 463-491 (1998)

34. Stokes, G.G.: On the theories of the internal friction of fluids in motion, and of the equilibrium and motion of elestic solids. Trans. Camb. Philos. Soc. 8, 287-305 (1845)

35. Swann, H.S.G.: The convergence with vanishing viscosity of nonstationary Navier-Stokes flow to ideal flow in $R^{3}$. Trans. Am. Math. Soc. 157, 373-397 (1971)

36. Temam, R.: Navier-Stokes Equations and Nonlinear Functional Analysis, 2nd edn. Society for Industrial and Applied Mathematics, Philadelphia (1995)

37. Thompson, P.A., Troian, S.M.: A general boundary condition for liquid flow at solid surface. Nature 389, 360-362 (1997)

38. Wang, X.: A Kato type theorem on zero viscosity limit of Navier-Stokes flows. Indiana Univ. Math. J. 50, 223-241 (2001). Special Issue Dedicated to Professors Ciprian Foias and Roger Temam (Bloomington, IN, 2000)

39. Wang, X., Wang, Y., Xin, Z.: Boundary layers in incompressible Navier-Stokes equations with Navier boundary conditions for the vanishing viscosity limit. Commun. Math. Sci. 8(4), 965-998 (2010)

40. Wang, L., Xin, Z., Zang, A.: Vanishing viscous limits for 3D Navier-Stokes equations with a Navierslip boundary condition. J. Math. Fluid Mech. 14(4), 791-825 (2012)

41. Xiao, Y.L., Xin, Z.P.: On the vanishing viscosity limit for the 3D Navier-Stokes equations with a slip boundary condition. Commun. Pure Appl. Math. LX, 1027-1055 (2007) 
42. Xiao, Y.L., Xin, Z.P.: Remarks on the vanishing viscosity limit for 3D Navier-Stokes equations with a slip boundary condition. Chin. Ann. Math. 32B(3), 321-332 (2011)

43. Xiao, Y., Xin, Z.: On 3D Lagrangian Navier-Stokes $\alpha$ model with a class of vorticity slip boundary conditions. J. Math. Fluid Mech. (2012). doi:10.1007/s00021-012-0110-5

44. Xiao, Y.L., Xin, Z.P.: A new boundary condition for the 3D Navier-Stokes equation and the vanishing viscosity limit. J. Math. Phys. 53, 115617 (2012)

45. Xin, Z., Yanagisawa, T.: Zero-viscosity limit of the linearized Navier-Stokes equations for a compressible viscous fluid in the half-plane. Commun. Pure Appl. Math. 52(4), 479-541 (1999) 